\title{
Seismic fluid identification using a nonlinear elastic impedance inversion method based on a fast Markov chain Monte Carlo method
}

\author{
Guang-Zhi Zhang ${ }^{1} \cdot$ Xin-Peng Pan ${ }^{1} \cdot{\text { Zhen-Zhen } \mathrm{Li}^{1} \cdot \text { Chang-Lu Sun }}^{1} \cdot$ \\ Xing-Yao Yin ${ }^{1}$
}

Received: 7 January 2015/Published online: 28 July 2015

(c) The Author(s) 2015. This article is published with open access at Springerlink.com

\begin{abstract}
Elastic impedance inversion with high efficiency and high stability has become one of the main directions of seismic pre-stack inversion. The nonlinear elastic impedance inversion method based on a fast Markov chain Monte Carlo (MCMC) method is proposed in this paper, combining conventional MCMC method based on global optimization with a preconditioned conjugate gradient (PCG) algorithm based on local optimization, so this method does not depend strongly on the initial model. It converges to the global optimum quickly and efficiently on the condition that efficiency and stability of inversion are both taken into consideration at the same time. The test data verify the feasibility and robustness of the method, and based on this method, we extract the effective pore-fluid bulk modulus, which is applied to reservoir fluid identification and detection, and consequently, a better result has been achieved.
\end{abstract}

Keywords Elastic impedance - Nonlinear inversion - Fast Markov chain Monte Carlo method · Preconditioned conjugate gradient algorithm - Effective pore-fluid bulk modulus

\section{Introduction}

Compared to amplitude versus offset (AVO) inversion based on common mid-point (CMP) gathers, elastic impedance inversion based on partial angle-stack gathers

Xin-Peng Pan

panxinpeng1990@gmail.com

1 School of Geosciences, China University of Petroleum (East China), Qingdao 266580, Shandong, China

Edited by Jie Hao has the advantages of high computational efficiency, high stability, high noise immunity, and low dependence on the quality of seismic data. This has been widely used in reservoir fluid identification and detection, and has become one of the main directions of pre-stack inversion (Downton 2005; Yin et al. 2014).

Connolly (1999) proposed the concept of elastic impedance on the basis of acoustic impedance for the first time. Cambois (2000) considered that the high noise immunity of elastic impedance could avoid "leakage" between the various AVO attributes generated by noise. This is more advantageous in the extraction of pre-stack parameters. Whitcombe (2002) first applied a new normalized form of elastic impedance to improve the stability of parameter extraction. Additionally, Martins (2006) and Cui et al. (2010) introduced P- and P-SV wave elastic impedance in weakly anisotropic media, respectively. In recent years, fluid indicators estimated from seismic data play important roles in reservoir characterization and prospect identification, so many methods, such as elastic impedance inversion, have been introduced to extract a variety of fluid factors directly to avoid the cumulative error generated by indirect combination of parameters in the process of reservoir fluid identification (Ma 2003; Peng et al. 2008; Zong et al. 2011, 2012; Yin et al. 2013b; Zhang et al. 2013; Chen et al. 2014a, b; Li et al. 2014). Goodway et al. (1997) proposed $\lambda \rho$ and $\mu \rho$ as fluid indicators. Russell et al. (2011) used $f$ as a fluid indicator based on the poroelastic theory. However, the sensitivity of these fluid indicators is dependent on the mixed effect of pore fluid and rock matrix. To improve the sensitivity of reservoir fluid identification, we use the effective pore-fluid bulk modulus as the fluid indicator, which is related only to pore fluid and may diminish the rock-matrix effect (Han and Batzle 2004; Yin and Zhang 2014). 
The present elastic impedance inversion techniques are mostly based on linear or quasi-linear inversion methods, not only losing the precision of inversion in the process of linearization but also strongly relying on the accuracy of initial model, while convergence to the global optimum is difficult in the usage of these techniques ( $\mathrm{Su}$ et al. 2014). However, the nature of most inverse problems is nonlinear and multi-extremum, so in terms of the complex pre-stack reservoir elastic parameter inversion, the development of elastic impedance with high efficiency and high stability based on the nonlinear inversion method has become more significant. We propose a nonlinear elastic impedance inversion method based on a fast Markov chain Monte Carlo (MCMC) method, and validate the feasibility and robustness of the method by testing the noise immunity of well data. Meanwhile, on the basis of two-phase medium theory for elastic impedance equation, we extract the effective pore-fluid bulk modulus from seismic data to apply to reservoir fluid identification and detection (Russell et al. 2003; Yin et al. 2013a).

\section{Fast MCMC method}

Hastings (1970) proposed an extended form of the Metropolis algorithm (Metropolis et al. 1953), the Metropolis-Hastings algorithm, laying the foundation for the development of the MCMC method. The MCMC method was first applied to fully nonlinear inverse problems by Malinverno (2002). Zhang et al. (2011a, b) studied post-stack and pre-stack seismic inversion methods based on the MCMC method. In recent years, the MCMC method has been applied to sample the posterior distribution of reservoir parameters for identifying reservoir lithology and fluid on the basis of a Bayesian framework (Bachrach et al. 2009; Grana and Rossa 2010; Rimstad and Omre 2010; Ulvmoen and Omre 2010; Ulvmoen et al. 2010; Rimstad et al. 2012). However, the conventional MCMC method has the defects of low computational efficiency and a low convergence rate toward the global optimum for multi-extremum inverse problems, so we propose a faster MCMC method than the conventional MCMC method in this paper.

\subsection{Metropolis-Hastings algorithm}

MCMC can sample posterior probability distribution converging to inverted parameters in Bayesian inference, and then make some statistical analysis of random samples to obtain useful properties of parameter posterior distribution.

The construction methods of the transition kernel play important roles in the MCMC method, including the Metropolis-Hastings (M-H) algorithm and Gibbs algorithm. We choose the $\mathrm{M}-\mathrm{H}$ algorithm, and after a sufficiently long iteration, stable Markov chains form, which can be used in making the statistical analysis for random samples of parameter posterior distribution, and which satisfy the detailed balance condition.

\subsection{Principle of fast MCMC method}

Conventional MCMC can be applied efficiently to nonlinear and single-extremum inverse problems, but it is difficult to converge to global optimums of multi-extremum inverse problems. Thus, we propose a fast MCMC method, which integrates an efficient optimization algorithm into the MCMC method to improve the convergence rate to the globally optimal solution, and greatly raises the computational efficiency of the MCMC method.

The PCG algorithm is considered an efficient and stable optimization algorithm (Stefano et al. 2013), so we combine the MCMC method based on the global optimal $\mathrm{M}-\mathrm{H}$ algorithm with the PCG algorithm equipped with local search ability. Overall, the central idea of the fast MCMC method proposed in this paper is the integration of the more efficient PCG algorithm in the process of random search in whole space based on the global optimal MCMC method. The method can search the solution of inverted parameters for fast objective function convergence, which saves the huge computational cost of large numbers of iterations compared to the conventional MCMC method. We call the more efficient algorithm the "fast MCMC method", and its specific process is shown in Fig. 1.

To test the feasibility of the fast MCMC method, we designed a simple bimodal probability density function (PDF) $p(x)=0.3 \times \mathrm{e}^{\alpha x^{2}}+0.7 \times \mathrm{e}^{\alpha(x-10)^{2}}$, and the estimated parameter is $\alpha$, whose true value is -0.2 . Figure 2 shows two inverted results of 250 iterations from the conventional MCMC method (blue solid line) and the fast MCMC method (red solid line), respectively. From Fig. 2, we find that the fast MCMC method converges to a global optimal solution quickly, but conventional MCMC method has not reached convergence condition after 250 


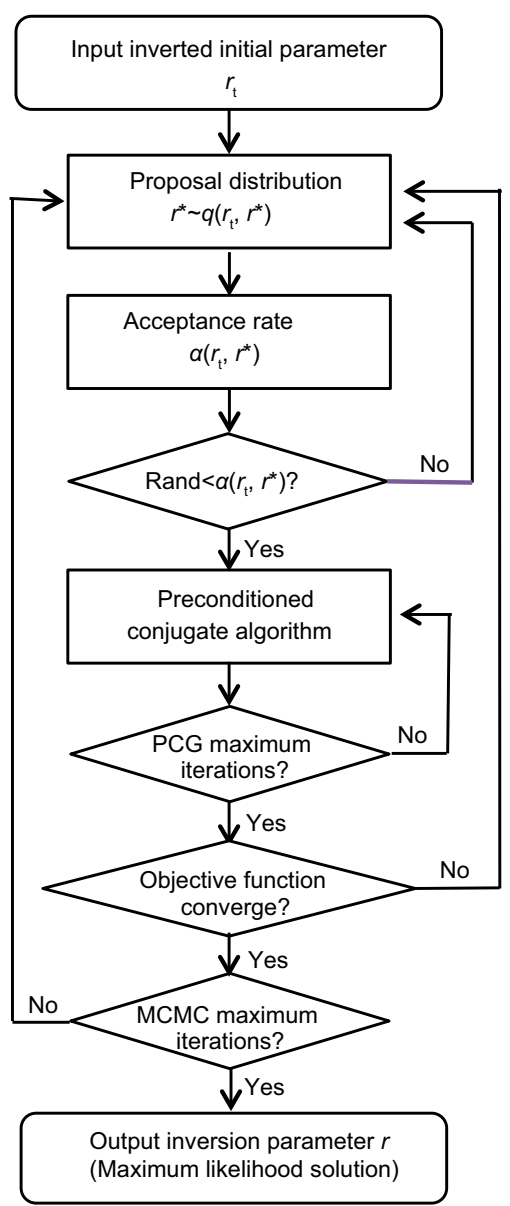

Fig. 1 Process of the fast MCMC method

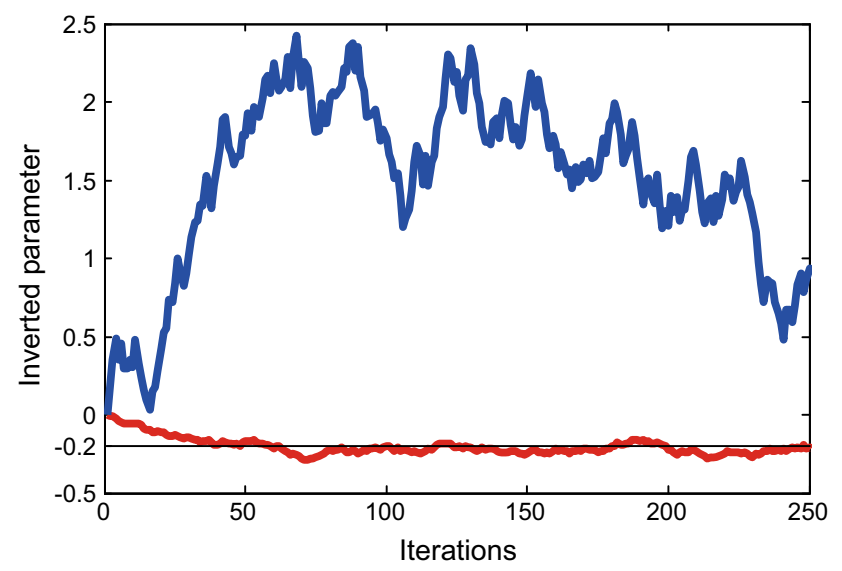

Fig. 2 Comparison of the fast MCMC result (red) and the conventional MCMC result (blue) within 250 iterations

iterations, verifying that the fast MCMC method is more efficient than conventional MCMC method to converge to a global optimum.

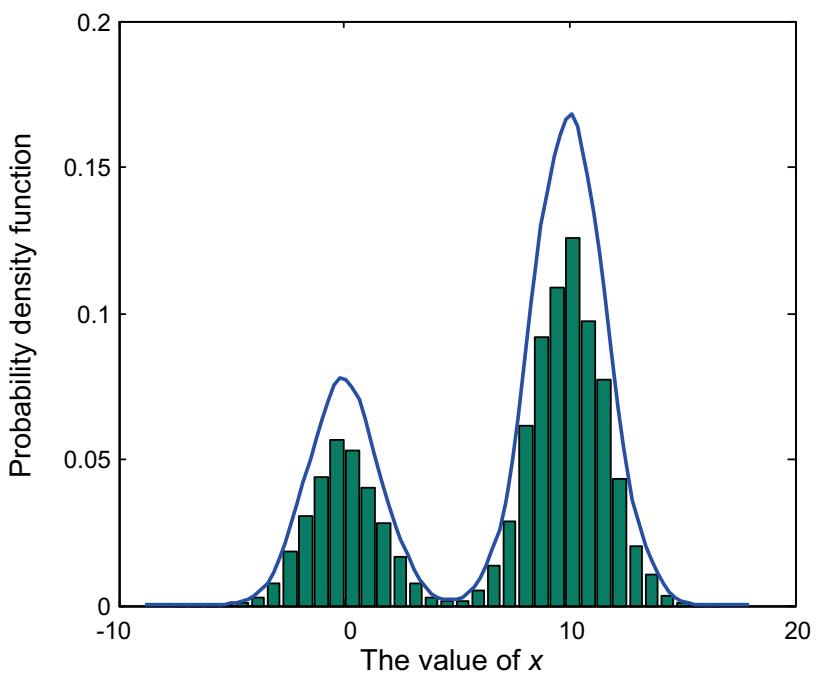

Fig. 3 Statistical characteristics of bimodal PDF sampled by the fast MCMC method

The maximum likelihood solution of unknown parameter $\alpha$ is regarded as the estimated value, and then, we sample the bimodal PDF $p(x)$. As shown in Fig. 3, the statistical characteristics of random samples sampled by the fast MCMC method are quite consistent with the characteristics of bimodal PDF, further verifying the feasibility and reliability of the fast MCMC method and laying the foundation for the fast algorithm of the nonlinear elastic impedance inversion method.

\section{Nonlinear elastic impedance inversion method based on the fast MCMC method}

The elastic inversion method used mostly now is the constrained sparse spike linear inversion method developed in the 1980 s, so we propose a nonlinear elastic impedance inversion method based on the fast MCMC method to improve the accuracy of elastic impedance inversion and the reliability of reservoir prediction and fluid identification.

\subsection{Elastic impedance equation based on two-phase medium theory}

To improve the reliability of reservoir pore-fluid discrimination, we choose the normalized elastic impedance equation on the basis of two-phase medium theory, which highlights the effective pore-fluid bulk modulus $K_{\mathrm{f}}$ (Yin et al. 2013a; Yin and Zhang 2014): 
$E I(\theta)=E I_{0}\left(\frac{K_{\mathrm{f}}}{K_{\mathrm{f} 0}}\right)^{a(\theta)}\left(\frac{f_{\mathrm{m}}}{f_{\mathrm{m} 0}}\right)^{b(\theta)}\left(\frac{\rho}{\rho_{0}}\right)^{c(\theta)}\left(\frac{\phi}{\phi_{0}}\right)^{d(\theta)}$,

where

$a(\theta)=\left(1-\frac{\gamma_{\mathrm{dry}}^{2}}{\gamma_{\text {sat }}^{2}}\right) \frac{\sec ^{2} \theta}{2}$

$b(\theta)=\frac{\gamma_{\mathrm{dry}}^{2}}{2 \gamma_{\mathrm{sat}}^{2}} \sec ^{2} \theta-\frac{4}{\gamma_{\mathrm{sat}}^{2}} \sin ^{2} \theta$

$c(\theta)=1-\frac{\sec ^{2} \theta}{2}$

$d(\theta)=\frac{\sec ^{2} \theta}{2}-\frac{\gamma_{\text {dry }}^{2}}{\gamma_{\text {sat }}^{2}} \sec ^{2} \theta+\frac{4}{\gamma_{\text {sat }}^{2}} \sin ^{2} \theta$,

and

$E I_{0}=K_{\mathrm{f}}^{-1 / 2} f_{\mathrm{m}} \phi^{-3 / 2} \rho^{1 / 2} \gamma_{\text {sat }}\left(\gamma_{\text {sat }}^{2}-\gamma_{\text {dry }}^{2}\right)^{1 / 2}$,

where $K_{\mathrm{f}}$ is the effective pore-fluid bulk modulus term, $f_{\mathrm{m}}=\phi \mu$ is the dry rock matrix term, $\rho$ is density, and $\phi$ is porosity value; $K_{\mathrm{f} 0}, f_{\mathrm{m} 0}, \rho_{0}$, and $\phi_{0}$ are the average effective pore-fluid bulk modulus, dry rock matrix term, density, and porosity value of well data; $E I_{0}$ is the normalization coefficient; $\theta$ is the average of the incident and refracted angles; $\gamma_{\text {dry }}^{2}$ and $\gamma_{\text {sat }}^{2}$ are the square of the P- to $\mathrm{S}$-wave velocities of the dry rock and saturated rock, respectively.

\subsection{Nonlinear elastic impedance inversion method based on the fast MCMC method}

Based on Bayes' theorem, the posterior probability density of inverted parameter $r$ is expressed as

$p(r \mid d) \propto p(r) \cdot p(d \mid r)$,

where $d$ is the seismic observation data, $r$ is the reflection coefficient sequence, $p(r \mid d)$ is the posterior probability of the reflection coefficient, $p(r)$ is the prior information, and $p(d \mid r)$ is a likelihood function.

Assuming seismic background noise obeys an independent Gaussian distribution with a zero mean and $\sigma_{n}^{2}$ variance, the seismic observation likelihood function can be expressed as

$p(d \mid I)=\frac{1}{\left(2 \pi \sigma_{n}^{2}\right)^{\frac{N}{2}}} \exp \left(-\sum \frac{(d-G r)^{\mathrm{T}}(d-G r)}{2 \sigma_{n}^{2}}\right)$.
Meanwhile, based on the assumption that the prior information obeys the Cauchy distribution to stand out weak reflection of the underground media, the prior information is expressed as

$p(r)=\frac{1}{\left(\pi \sigma_{r}\right)^{M}} \prod_{i=1}^{M}\left(\frac{1}{1+r^{2} / \sigma_{r}^{2}}\right)$.

To obtain the posterior probability distribution $p(r \mid d)$, we apply the Metropolis-Hastings algorithm to generate stable Markov chains converging to inverted parameter $r$.

However, the conventional MCMC method results in huge computational cost and there is likely to be instability in the inverted results. Therefore, having taken the computational efficiency and inversion stability into account, we apply the fast MCMC method (shown as Fig. 1) to generate stable Markov chains converging to the posterior probability density distribution of inverted parameter and invert the elastic impedance using a nonlinear method based on the fast MCMC method to identify fluid.

In the Metropolis-Hastings algorithm, we choose a symmetrical distribution satisfying a symmetric random walk as the proposal distribution of the algorithm, so the proposal distribution and acceptance rate are expressed as

$q\left(r_{t}, r *\right) \sim U\left(r_{t}-\right.$ delta,$r_{t}+$ delta $)$

and

$\alpha\left(r_{t}, r *\right)=\min \left\{1, \frac{\pi(r *) q\left(r *, r_{t}\right)}{\pi\left(r_{t}\right) q\left(r_{t}, r *\right)}\right\}=\min \left\{1, \frac{\pi(r *)}{\pi\left(r_{t}\right)}\right\}$.

In the PCG algorithm, on the Bayesian framework, we construct the objective function expressed as

$$
\begin{aligned}
J(r)= & (d-G r)^{\mathrm{T}}(d-G r)+\mu \sum_{i=1}^{M} \ln \left(1+r^{2} / \sigma_{r}^{2}\right) \\
& +\alpha(\eta-C r)^{\mathrm{T}}(\eta-C r),
\end{aligned}
$$

where $\mu=2 \frac{\sigma_{n}^{2}}{\sigma_{r}^{2}}$ is the sparse constraint factor, and the larger its value is, the more sparse the reflection coefficient is; the last term in the equation is the elastic impedance constraint term, $C$ and $\eta$ are integral operator matrix and relative impedance value, respectively, and $\alpha$ is the elastic impedance constraint factor, and the larger the value is, the 
more stable and accurate the inverted results are. Optimizing the objective function, we get the ultimate equation expressed as

$\left(G^{\mathrm{T}} G+\mu Q+\alpha C^{\mathrm{T}} C\right) r=\left(G^{\mathrm{T}} d+\alpha C^{\mathrm{T}} \eta\right)$,

where $Q=\operatorname{diag}\left[\cdots, \frac{1}{\left(1+r_{i}^{2} / \sigma_{r}^{2}\right)^{2}}, \cdots\right]$, and it adds the denominator squared term to reduce the effect of strength contrast and to highlight weak reflection of the underground media, and it can be termed the modified Cauchy constraint (Alemie and Sacchi 2011). Eventually, we apply the fast MCMC method to invert the reflection coefficient of different angles based on the convergence judgment of Eq. (7), and then, we obtain the elastic impedance of different angles by using the path integral method or the recursion method.

\subsection{Direct extraction of the effective pore-fluid bulk modulus parameter}

Expecting to apply elastic impedance volume of at least four angles to extract the effective pore-fluid bulk modulus directly following Eq. (1), we must turn the equation into a log-domain equation at first, and then obtain 16 fitted regression coefficients $\left(a\left(\theta_{i}\right), b\left(\theta_{i}\right), c\left(\theta_{i}\right), d\left(\theta_{i}\right), i=1,2\right.$, $3,4)$ via the least square method or the singular value decomposition method by using the elastic impedance data and well $\log$ data from nearby wells, and finally the inverted elastic impedance data volume of four angles is put into the equation expressed as

\subsection{Process of fluid identification using the nonlinear elastic impedance inversion method based on the fast MCMC method}

Synthesizing the research and analysis above, we conclude that the whole process of fluid identification using the nonlinear elastic impedance inversion method based on the fast MCMC method proposed in this paper is as follows:

1. Pretreatment of pre-stack seismic data and well log data;

2. Accurate extraction of four angle wavelets from prestack seismic data;

3. Inversion of elastic impedance of four angles based on the fast MCMC method by using four abstracted anglestack seismic gathers and four angles extracted from seismic data from nearby wells;

4. Extraction of effective pore-fluid bulk modulus from inverted elastic impedance of four angles on the basis of two-phase medium theory for the elastic impedance equation as the representation of reservoir pore-fluid information;

5. Application of extracted effective pore-fluid bulk modulus to identify fluid and predict reservoir parameters.

\section{Model test}

To test the feasibility of fluid identification using elastic impedance based on the fast MCMC method, we carry

$$
\left\{\begin{array}{l}
\ln \frac{E I\left(t, \theta_{1}\right)}{E I_{0}}=a\left(\theta_{1}\right) \ln \frac{K_{\mathrm{f}}(t)}{K_{\mathrm{f} 0}}+b\left(\theta_{1}\right) \ln \frac{f_{\mathrm{m}}(t)}{f_{\mathrm{m} 0}}+c\left(\theta_{1}\right) \ln \frac{\rho(t)}{\rho_{0}}+d\left(\theta_{1}\right) \ln \frac{\phi(t)}{\phi_{0}} \\
\ln \frac{E I\left(t, \theta_{2}\right)}{E I_{0}}=a\left(\theta_{2}\right) \ln \frac{K_{\mathrm{f}}(t)}{K_{\mathrm{f} 0}}+b\left(\theta_{2}\right) \ln \frac{f_{\mathrm{m}}(t)}{f_{\mathrm{m} 0}}+c\left(\theta_{2}\right) \ln \frac{\rho(t)}{\rho_{0}}+d\left(\theta_{2}\right) \ln \frac{\phi(t)}{\phi_{0}} \\
\ln \frac{E I\left(t, \theta_{3}\right)}{E I_{0}}=a\left(\theta_{3}\right) \ln \frac{K_{\mathrm{f}}(t)}{K_{\mathrm{f} 0}}+b\left(\theta_{3}\right) \ln \frac{f_{\mathrm{m}}(t)}{f_{\mathrm{m} 0}}+c\left(\theta_{3}\right) \ln \frac{\rho(t)}{\rho_{0}}+d\left(\theta_{3}\right) \ln \frac{\phi(t)}{\phi_{0}} \\
\ln \frac{E I\left(t, \theta_{4}\right)}{E I_{0}}=a\left(\theta_{4}\right) \ln \frac{K_{\mathrm{f}}(t)}{K_{\mathrm{f} 0}}+b\left(\theta_{4}\right) \ln \frac{f_{\mathrm{m}}(t)}{f_{\mathrm{m} 0}}+c\left(\theta_{4}\right) \ln \frac{\rho(t)}{\rho_{0}}+d\left(\theta_{4}\right) \ln \frac{\phi(t)}{\phi_{0}}
\end{array}\right.
$$

So we can obtain the effective pore-fluid bulk modulus at any sampling point. out the feasibility and noise immunity test of a well in one work area in the eastern China. The target reservoir 

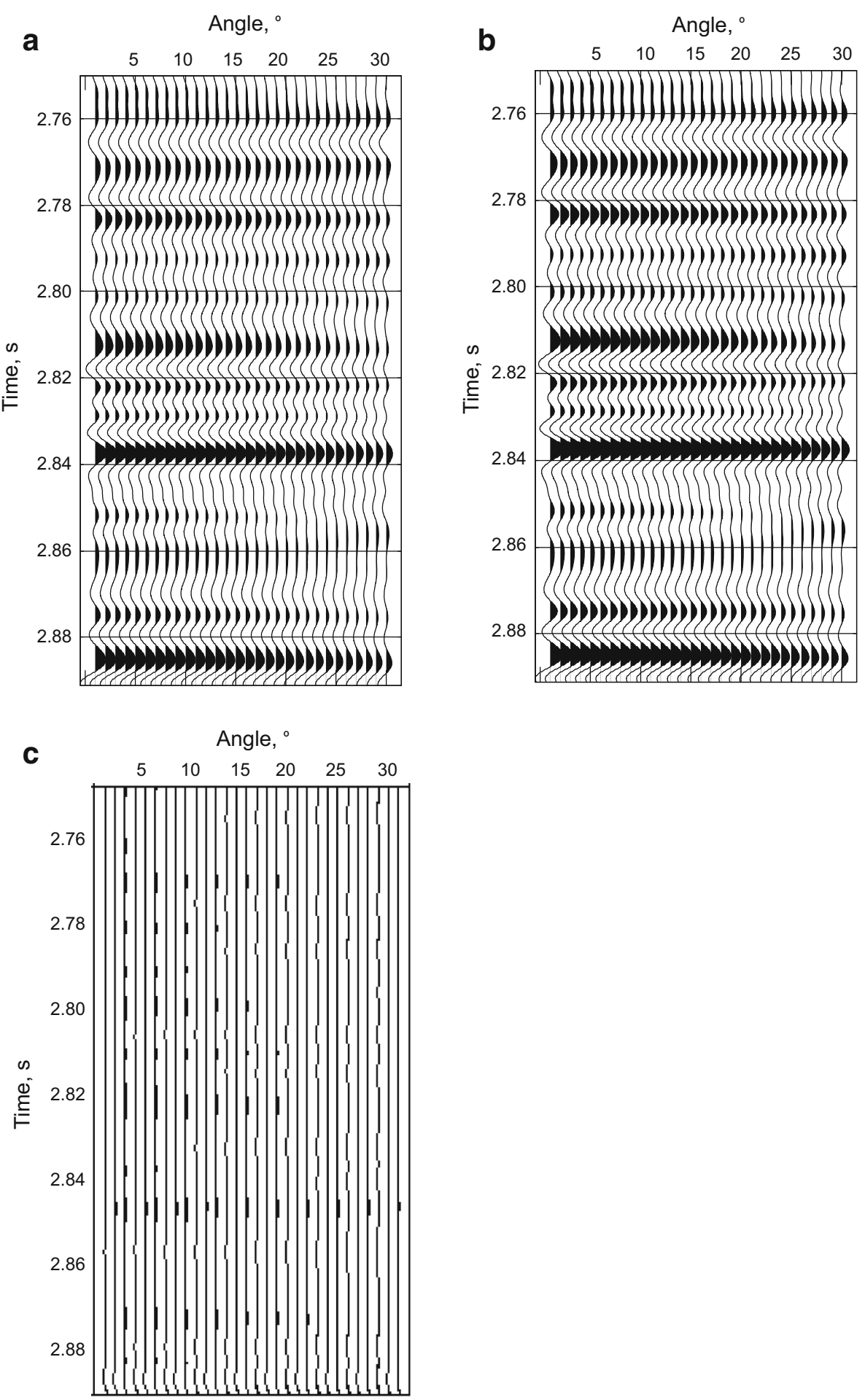

Fig. 4 Comparison of synthetic angle gathers using well log data and gathers by using inverted results in a noise-free situation. a synthetic angle gathers by using well data, b synthetic angle gathers by using inverted results in noise-free situation, c residual error between two synthetic angle gathers 


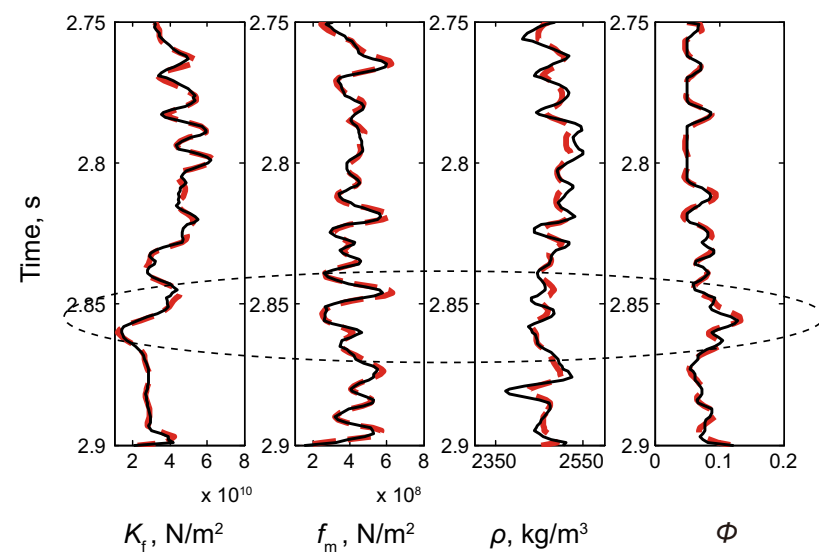

Fig. 5 Inverted parameter results from using the nonlinear elastic impedance inversion method based on the efficient MCMC in a noisefree situation (black and red solid lines indicate true values and inverted results, respectively; black dashed ellipse shows the oilbearing reservoir from logging interpretation)

is a clastic reservoir at about $2.85 \mathrm{~s}$ in the seismic profile.

As shown in Figs. 4a and 6a, we apply well log data to make a synthetic seismogram in noise and noise-free situations, and then generate high-accuracy and high-resolution elastic impedance of four angles based on the fast MCMC method for the direct extraction of effective porefluid bulk modulus parameters, testing the feasibility and noise immunity of the method.

From Fig. 5, we find that inverted effective pore-fluid bulk modulus, dry rock matrix term, density, and porosity values in the noise-free situation are consistent with the true values, and synthetic angle gathers using these inverted results produce fewer errors compared with real angle gathers, so the inverted parameters can obviously reflect the major characteristics of the oil-bearing reservoir, agreeing with the results of oil-bearing reservoir from logging interpretation.

From Fig. 7, we find that when adding random noise in $\mathrm{SNR}=3$ to the synthetic seismograms, inverted effective pore-fluid modulus, dry rock matrix term, density, and porosity values are also consistent with the true values, reflecting the major characteristics of the oil-bearing reservoir well. Therefore, it validates the robustness and noise immunity of the nonlinear elastic impedance inversion method based on the fast MCMC in fluid identification, and the inverted parameters are relatively accurate, which can reflect the major characteristics of the oilbearing reservoir well and be applied to identify fluid and predict reservoirs.

\section{Application of real seismic data}

The real work area is selected from an exploration area in eastern China, and the target is a clastic reservoir. As shown in Fig. 8, the logging interpretation results indicate that the clastic reservoir at $2.85 \mathrm{~s}$ shows an oil layer with thickness up to $13 \mathrm{~m}$. To verify the application effect of the method of fluid identification using the nonlinear elastic impedance inversion based on the fast MCMC method, we apply this method to real seismic data.

First of all, we invert elastic impedance based on the fast MCMC method using angle-stack seismic profiles with four different angles, and the inverted elastic impedance profiles of four angles are shown in Fig. 9, in which the well $\operatorname{logs}$ are elastic impedance curves of four different angles. Then, we extract the effective pore-fluid bulk modulus based on inverted elastic impedance data volume of four angles directly, and apply them to fluid identification and reservoir prediction. With the two effective porefluid bulk modulus logging curves in Fig. 10, the figure shows the extracted effective pore-fluid bulk modulus profile and enlarged partial profile.

From Fig. 9, we find that the inverted elastic impedance profiles based on the fast MCMC method with four different angles are highly precise, which agree with the results from the oil-bearing reservoir logging interpretation.

Figure 10 shows that the extracted effective pore-fluid bulk modulus is also consistent with the logging interpretation results, presenting low values and reflecting the characteristics of the oil-bearing reservoir, so it further 

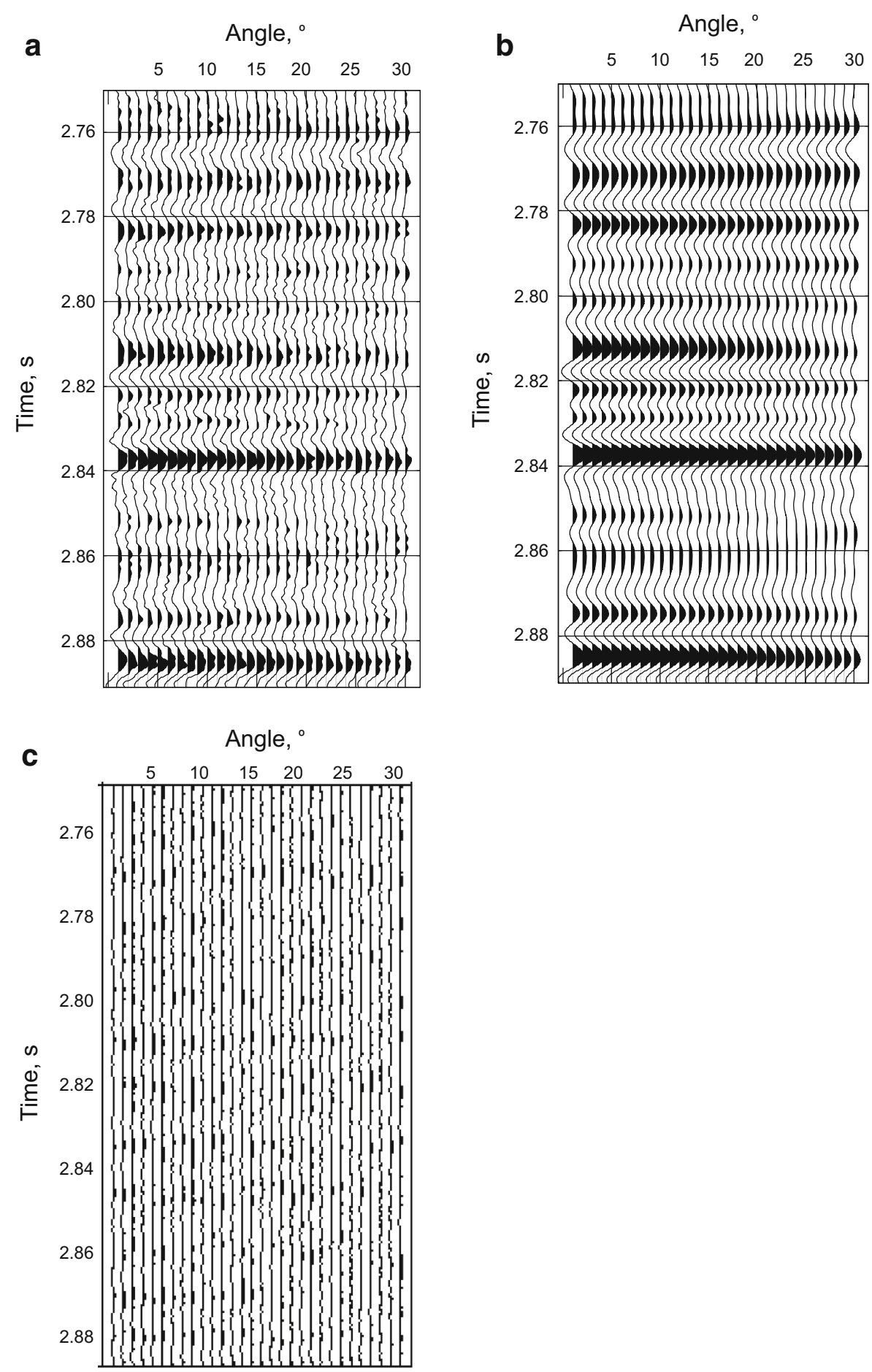

Fig. 6 Comparison of synthetic angle gathers using well data and gathers by using inverted results in a noise situation (SNR $=3$ ). a synthetic angle gathers using well data, $\mathbf{b}$ synthetic angle gathers by using inverted results in the noise situation, $\mathbf{c}$ residual error between two synthetic angle gathers 


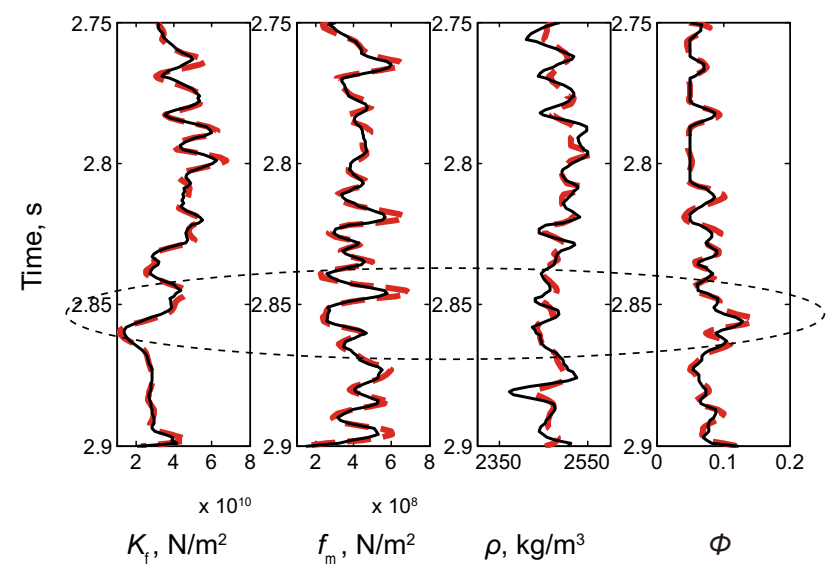

Fig. 7 Inverted parameter results from the nonlinear elastic impedance inversion method based on the efficient MCMC in the noise situation $(\mathrm{SNR}=3)$ (black and red solid lines indicate true values and inverted results respectively; the black dashed ellipse shows the oil-bearing reservoir from the logging interpretation) validates the reservoir fluid detection and identification from seismic data by using the nonlinear elastic impedance inversion method based on the fast MCMC method, which is promising in practical applications.

\section{Conclusions}

We introduce a more reliable fluid identification method using nonlinear elastic impedance inversion based on the fast MCMC in this paper, and the fast MCMC method is the key of foundation for the nonlinear elastic impedance inversion algorithm. Based on the Bayesian framework, we can identify the reservoir hydrocarbon more accurately owing to the more sensitive reservoir fluid indicator, that is the effective pore-fluid bulk modulus, and this method may improve the reliability and stability of fluid identification. Application research based on actual logging and
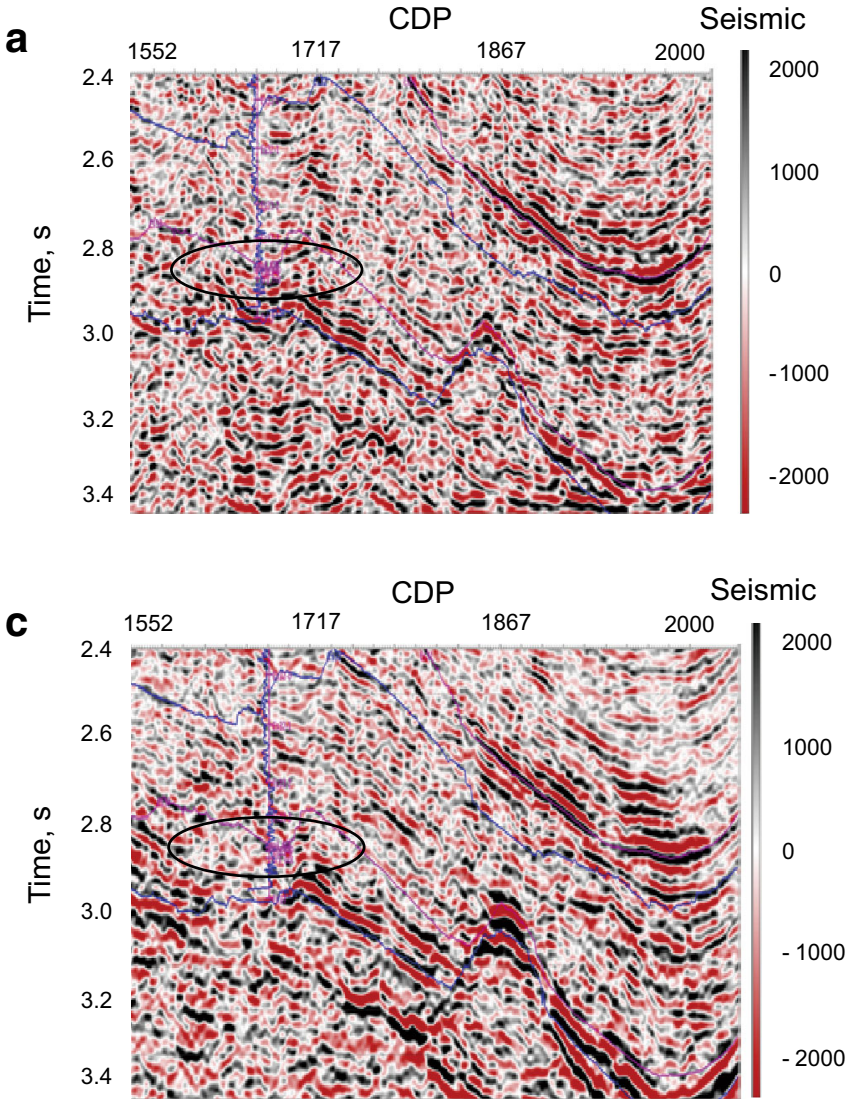
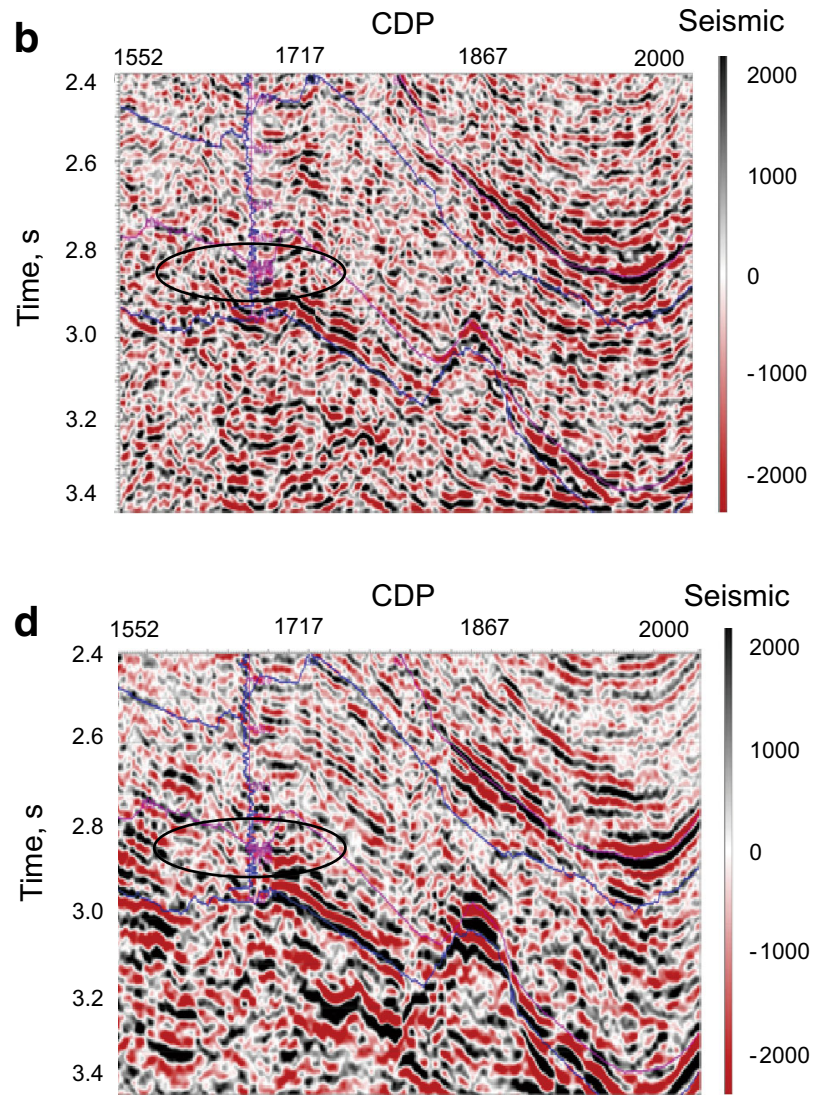

Fig. 8 Angle-stack seismic profiles with four different angles. a $6^{\circ}\left(3^{\circ}-9^{\circ}\right)$ b $12^{\circ}\left(9^{\circ}-15^{\circ}\right)$ c $18^{\circ}\left(15^{\circ}-21^{\circ}\right)$ d $24^{\circ}\left(21^{\circ}-27^{\circ}\right)$ 

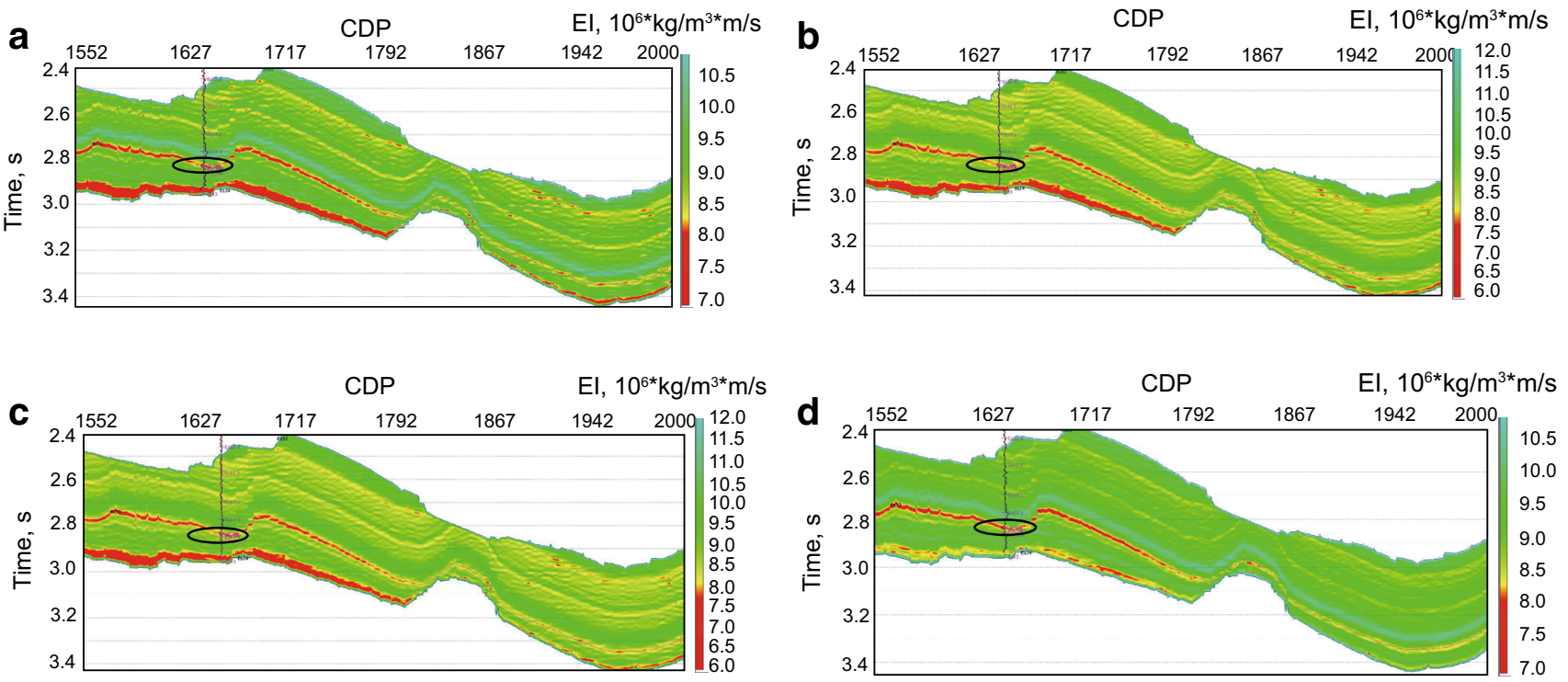

Fig. 9 Inverted elastic impedance profiles based on the fast MCMC method with four different angles. a $6^{\circ}$ elastic impedance profile, $\mathbf{b} 12^{\circ}$ elastic impedance profile, c $18^{\circ}$ elastic impedance profile, $\mathbf{d} 24^{\circ}$ elastic impedance profile

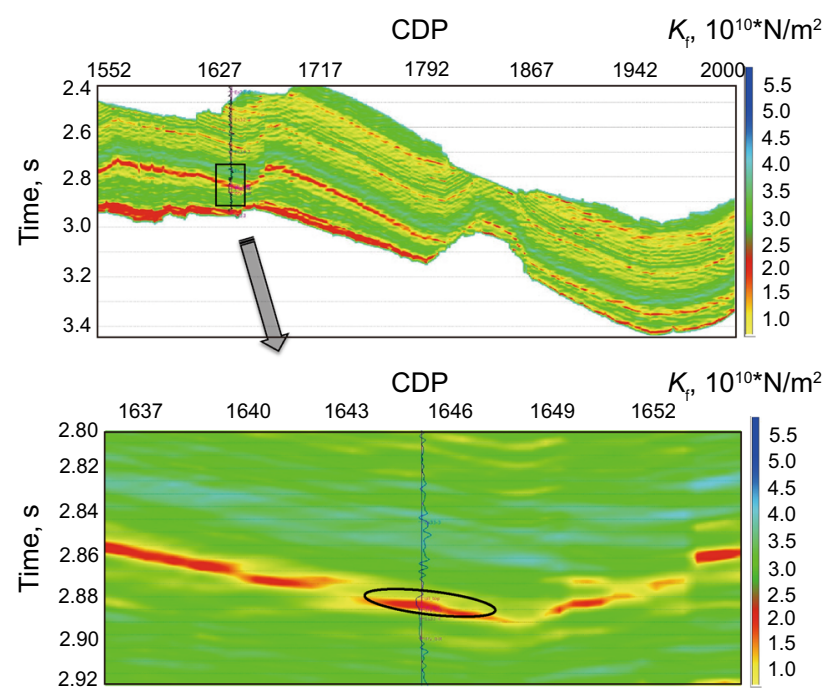

Fig. 10 Extracted effective pore-fluid bulk modulus profile and enlarged partial profile

seismic data shows that the nonlinear elastic impedance fluid identification method based on the fast MCMC is a practical method for reservoir fluid identification. However, the method has a limitation of obtaining only one inverted result of the effective pore-fluid bulk modulus, and may lack the uncertainty evaluation about reservoir fluid identification. So further in-depth research and analysis is needed, and we are intending to continue our research in this field.
Acknowledgments We would like to express our gratitude to the sponsorship of the National Basic Research Program of China (973 Program, 2013CB228604, 2014CB239201) and the National Oil and Gas Major Projects of China (2011ZX05014-001-010HZ, 2011ZX05014-001-006-XY570) for their funding of this research. We also thank the anonymous reviewers for their constructive suggestions.

Open Access This article is distributed under the terms of the Creative Commons Attribution 4.0 International License (http://crea tivecommons.org/licenses/by/4.0/), which permits unrestricted use, distribution, and reproduction in any medium, provided you give appropriate credit to the original author(s) and the source, provide a link to the Creative Commons license, and indicate if changes were made.

\section{References}

Alemie W, Sacchi MD. High-resolution three-term AVO inversion by means of a Trivariate Cauchy probability distribution. Geophysics. 2011;76(3):R43-55.

Bachrach R, Sengupta M, Salama A, et al. Reconstruction of the layer anisotropic elastic parameter and high resolution fracture characterization from P-wave data: a case study using seismic inversion and Bayesian rock physics parameter estimation. Geophys Prospect. 2009;57(2):253-62.

Cambois G. AVO inversion and elastic impedance. 70th annual international meeting, SEG, Expanded Abstract. 2000. pp. 142-5.

Chen HZ, Yin XY, Gao CG, et al. AVAZ inversion for fluid factor based on fracture anisotropic rock physics theory. Chin J Geophys. 2014a;57(3):968-78 (in Chinese).

Chen HZ, Yin XY, Zhang JQ, et al. Seismic inversion for fracture rock physics parameters using azimuthally anisotropic elastic impedance. Chin J. Geophys. 2014b;57(10):3431-41 (in Chinese).

Connolly P. Elastic impedance. Lead Edge. 1999;18(4):438-52. 
Cui J, Han LG, Liu QK, et al. P-SV wave elastic impedance and fluid identification factor in weakly anisotropic media. Appl Geophys. 2010;7(2):135-42.

Downton JE. Seismic parameter estimation from AVO inversion. Ph.D. Thesis, University of Calgary. 2005.

Goodway WN, Chen T, Downton J. Improved AVO fluid detection and lithology discrimination using Lamé petrophysical parameters; " $\lambda$ ", " $\mu$ ", and " $\lambda / \mu$ fluid stack", from $\mathrm{P}$ and $\mathrm{S}$ inversions. 67th annual international meeting, SEG, Expanded Abstracts. 1997. pp. 183-6.

Grana D, Rossa ED. Probabilistic petrophysical-properties estimation integrating statistical rock physics with seismic inversion. Geophysics. 2010;75(3):O21-37.

Han DH, Batzle ML. Gassmann's equation and fluid-saturation effects on seismic velocities. Geophysics. 2004;69(2):398-405.

Hastings WK. Monte Carlo sampling methods using Markov chains and their applications. Biometrika. 1970;57(1):97-109.

Li C, Yin XY, Zhang GZ, et al. Two-term elastic impedance inversion based on the incident-angle approximation. Chin J Geophys. 2014;57(10):3442-52 (in Chinese).

Ma JF. Forward modeling and inversion method of generalized elastic impedance in seismic exploration. Chin J Geophys. 2003;46(1):118-24 (in Chinese).

Malinverno A. Parsimonious Bayesian Markov chain Monte Carlo inversion in a nonlinear geophysical problem. Geophys $\mathbf{J}$ Int. 2002;151(3):675-88.

Martins JL. Elastic impedance in weakly anisotropic media. Geophysics. 2006;71(3):D73-83.

Metropolis N, Rosenbluth AW, Rosenbluth MN, et al. Equations of state calculations by fast computing machines. J Chem Phys. 1953;21(6):1087-92.

Peng ZM, Li YL, Wu SH, et al. Discriminating gas and water using multi-angle extended elastic impedance inversion in carbonate reservoirs. Chin J Geophys. 2008;51(3):881-5 (in Chinese).

Rimstad K, Omre H. Impact of rock-physics depth trends and Markonv random fields on hierarchical Bayesian lithology/fluid prediction. Geophysics. 2010;75(4):93-108.

Rimstad K, Avseth P, Omre H. Hierarchical Bayesian lithology/ prediction: a North Sea case study. Geophysics. 2012;77(2): B39-85.

Russell BH, Hedlin K, Hilterman FJ, et al. Fluid-property discrimination with AVO: a Biot-Gassmann perspective. Geophysics. 2003;68(1):29-39.

Russell B, Gray D, Hampson D. Linearized AVO and poroelasticity. Geophysics. 2011;76(3):C19-29.
Stefano MD, Andreasi FG, Secchi A. Towards preconditioned nonlinear conjugate gradient for generic geophysical inversions. 83th annual international meeting, SEG, Expanded Abstract. 2013. pp. 3226-30.

Su JL, Mi H, Wang YC, et al. Non-linear elastic impedance inversion method supported by vector machines. Oil Geophys Prospect. 2014;39(4):751-8 (in Chinese).

Ulvmoen M, Omre H. Improved resolution in Bayesian lithology/fluid inversion from pre-stack seismic data and well observations: part 1-Methodology. Geophysics. 2010;75(2):R21-35.

Ulvmoen M, Omre H, Buland A. Improved resolution in Bayesian lithology/fluid inversion from pre-stack seismic data and well observations: part 2-real case study. Geophysics. 2010;75(2): B73-82.

Whitcombe DN. Elastic impedance normalization. Geophysics. 2002;67(1):60-2.

Yin XY, Li C, Zhang SX. Seismic fluid discrimination based on twophase media theory. J China Univ Petrol. 2013a;37(5):38-43 (in Chinese).

Yin XY, Zhang SX, Zhang FC, et al. Two-term elastic impedance inversion and Russell fluid factor direct estimation method for deep reservoir fluid identification. Chin J Geophys. 2013b;56(7): 2378-90 (in Chinese).

Yin XY, Cao DP, Wang BL, et al. Research progress of fluid discrimination with pre-stack seismic inversion. Oil Geophys Prospect. 2014. 49(1):22-34, 46 (in Chinese).

Yin XY, Zhang SX. Bayesian inversion for effective pore-fluid bulk modulus based on fluid-matrix decoupled amplitude variation with offset approximation. Geophysics. 2014;79(5):R221-32.

Zhang GZ, Chen HZ, Wang Q, et al. Estimation of S-wave velocity and anisotropic parameter using fractured carbonate rock physics model. Chin J Geophys. 2013;56(5):1707-15 (in Chinese).

Zhang GZ, Wang DY, Yin XY, et al. Study on pre-stack seismic inversion using Markov chain Monte Carlo. Chin J Geophys. 2011a;54(11):2926-32 (in Chinese).

Zhang GZ, Wang DY, Yin XY. Seismic parameter estimated using Markov chain Monte Carlo method. Oil Geophys Prospect. 2011b;46(4):605-9 (in Chinese).

Zong ZY, Yin XY, Wu GC. Fluid identification method based on compressional and shear modulus direct inversion. Chin $\mathrm{J}$ Geophys. 2012;55(1):284-92 (in Chinese).

Zong ZY, Yin XY, Zhang FC. Elastic impedance Bayesian inversion for Lamé parameters extraction. Oil Geophys Prospect. 2011; 46(4):598-604, 609 (in Chinese). 